\title{
Partial discharge characterisation in liquid nitrogen composite systems
}

\author{
D J Swaffield*, P L Lewin, Y Tian, G Chen and S G Swingler \\ University of Southampton, The Tony Davis High Voltage Laboratory, Electrical Power Engineering Research Group \\ Electronics and Computer Science, Highfield, Southampton, SO17 1BJ, UK \\ *djs@ecs.soton.ac.uk
}

\begin{abstract}
Interest in superconductors for use in the transmission and distribution of power has grown since the advent of High Temperature Superconductors (HTS). The benefits of reduced ohmic losses leading to greater operational efficiency appear attractive to power transmission and distribution companies. Potentially this technology will increase power transfer for the same or lower voltage and for the same or smaller physical dimensions. For the successful employment of this technology in the field, power utilities must be convinced of the operational reliability of designs. Many of these designs make use of liquid nitrogen as the system coolant and also as a dielectric fluid. It is therefore vital that liquid nitrogen be well characterised as a dielectric medium. Point-plane sample geometries have been studied for ac applied electric fields to extend understanding of pre-breakdown phenomena and density change streamer effects in liquid nitrogen composite insulation systems. A technique for time correlated measurement of partial discharge activity and density change streamer image recording has been employed to investigate the partial discharge activity when a highly divergent electric field is applied for multiple ac cycles. Example highspeed images are given showing the effects of streamer discharges experienced by the liquid both during and after the partial breakdown of the insulation. Work has been performed to characterise the partial discharge activity in composite solidliquid nitrogen insulation systems for different solid dielectrics resulting from the application of a $50 \mathrm{~Hz}$ highly divergent electric field. $\varphi q n$ analysis of ac results has been completed and characterisation of partial discharge behaviour for each system is presented and discussed.
\end{abstract}

\section{INTRODUCTION}

Within existing prototype designs for High Temperature Superconducting (HTS) equipment, liquid nitrogen is employed in the dual role as coolant and as a dielectric. Liquid nitrogen has a high dielectric strength, comparable to dielectric liquids at room temperature [1]. Liquid nitrogen breakdown data displays volume and area effects and are dependant on other parameters including purity, electrode material and surface condition [2]. Due to a narrow liquid temperature range the cryogenic liquid insulation suffers from the increased potential of density change to vapour. The increase in vapour in the insulation gap has been seen to reduce the breakdown voltage. Vapour can be introduced into the bulk liquid by nucleate boiling from conductors undergoing quench, due for example, to constraints in the design of an ambient to cryogenic current lead-through. Alternatively vapour may be introduced from electrical discharges into the liquid as shown in this experiment.
Partial discharge (pd) under ac conditions has previously been examined in [3] for a triple junction, and [4] for point plane. Point-plane samples are used extensively to produce a strongly divergent field. To better understand the pre-discharge phenomena under ac conditions partial discharge is characterised for $50 \mathrm{~Hz}$ ac voltage applied to a point-plane geometry.

\section{EXPERIMENTAL SETUP}

The experimental apparatus and the technique used for synchronous electrical partial discharge signal and image capture is detailed in [5]. The sample consists of a tungsten needle with a radius of $5.0 \pm 1.6 \mu \mathrm{m}$ a $20^{\circ}$ apex angle and a shank diameter of $1 \mathrm{~mm}$. This is earthed and positioned below a plane electrode covered with the solid insulator barrier. The three materials studied are $3 \mathrm{~mm}$ thick PTFE and $4 \mathrm{~mm}$ thick plates of two grades of Glass Reinforced Resin (GRR), Tufnol ${ }^{\circledR}$ Grade10G/40 and Tufnol ${ }^{\circledR}$ Grade 6 F40. The liquid nitrogen filled gap between needle tip was set to $3 \mathrm{~mm}$ for the PTFE and 6F/40 grade GRR and $2.6 \mathrm{~mm}$ for the $10 \mathrm{G} / 40$ grade GRR. The test sample is enclosed within a purpose built cryostat that allows application of voltage, pd free to $50 \mathrm{kV}$ and controlled temperature and pressure. The two GRR grades are comprised of multiple layers of glass fibre weave impregnated with resin. Both grades are mechanically stable over a large temperature range, however the PTFE required clamping to extra supports to prevent warping when cooled to the temperature of the liquid nitrogen. Following vacuuming of the cryostat inner vessel to remove moisture, it was filled with commercial grade liquid nitrogen.

\section{Experimental Procedure}

AC voltage and pressure are used as the working variables in this study. The pressure is first set by supplying helium gas into the top of the cryostat test chamber. AC voltage was then increased until the desired level was reached and recording begun. The camera was triggered simultaneously with the digital storage oscilloscope (dso) connected to capture pd activity measured via a Robinson pd detector Type700. The dso captured pd data for 200-250 cycles of applied ac voltage at $50 \mathrm{~Hz}$ power frequency. In addition pd signals have been correlated to images of density change streamers resulting from discharges, Figures 1 to 3 . These images were captured 
at 5000 frames per second (fps) using a high frequency pulse laser with a pulse duration of 30ns to stroboscopically backlight the needle sample. A microscope lens with long working distance was used to magnify the density charge streamer images. Images captured are 512 by 512 pixels, which with a pixel viewing an area of $2.42 \mu \mathrm{m}$ square equates to a full frame size of $1.24 \mathrm{~mm}$ square.

Since it is not possible to measure the true charge at a discharge site the electrical circuit was calibrated prior to use. This was performed by employing a standard technique; injecting pulses of known charge and measuring the output signal seen on the pd detector and recorded on the dso. This allows a measure of "apparent charge" for each density charge streamer recorded.

A typical approach when attempting to calculate the electric stress at the needle tip is to model the electrode geometry as a hyperboloid to plane. The calculation is simplified by two assumptions; 1) that the needle geometry is regular and is close to a hyperboloid in geometry. 2) That there are no space charge effects. This may be the case prior to the initial discharge but is unlikely to be correct after the generation of vapour and deposition of charge into the liquid and onto the solid insulation surface. The calculation of stress is further complicated for this sample by the presence of the solid insulation plates with additional space and surface charge effects.

\section{Analysis, $\varphi$ q n plotting}

The pd data captured have been analysed using $\varphi$ q n plotting, Figures 4 to 7 , to provide a useful visual representation of the results relating phase, cumulative number and charge magnitude of pd. Raw data are recorded as phase locations and amplitudes. The $50 \mathrm{~Hz}$ power cycle, period $20 \mathrm{~ms}$, is divided into 200 phase windows and 100 charge amplitude divisions. Cumulative numbers of discharges falling into these windows are calculated and plotted to provide a diagrammatical representation of partial discharge and allows comparison of activity under different test conditions. Since the zero crossing detection circuit has a delay that may be up to $11 \mu \mathrm{s}$ and a single discharge pulse may have a pulse width of up to $40 \mu$ s at the dso. The combined error may therefore be as much as $51 \mu \mathrm{s}$, equating to a 0.918 degrees phase shift. However this is less than one phase window width of 1.8 degrees. Therefore, over the whole cycle errors will not significantly influence the shape of the $\varphi \mathrm{q}$ n plot.

\section{RESULTS}

Two types of density change streamers have been observed, classified as "bush-like" and "filamentary". Bush-like streamers occur during the positive half cycle, i.e. when the needle is at a lower potential with respect to the plane electrode.
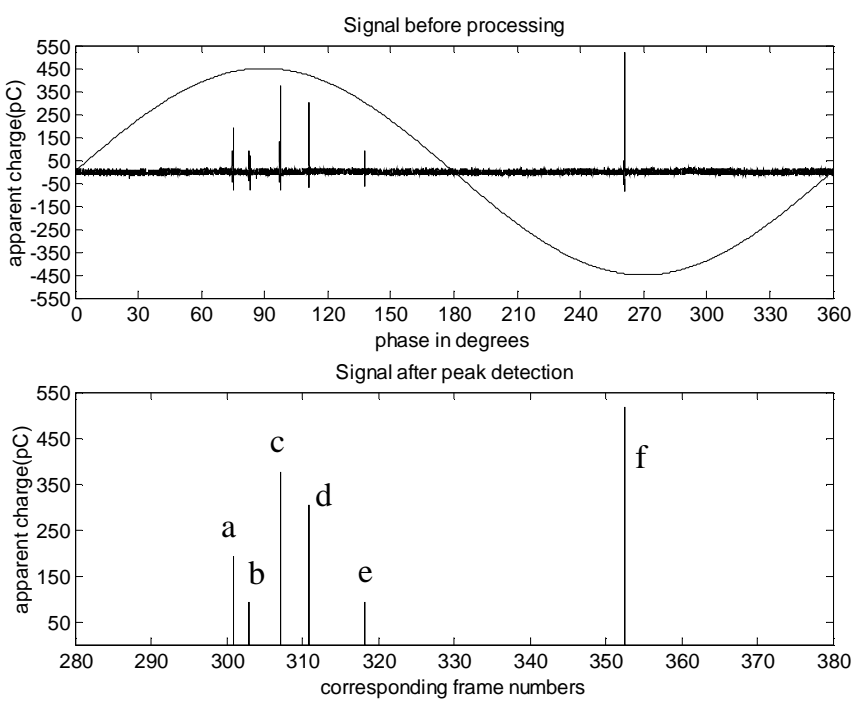

Figure 1, partial discharge signal at $18.28 \mathrm{kV}, 76.0 \pm 1 \mathrm{~K}$

Typical bush-like streamers are shown in figure 2. In this half-cycle it is usual that multiple discharges occur, the vapour from one discharge often remains in the gap while a subsequent discharge event occurs. Filamentary streamers appear in shape as single or multi-strand discharges with well defined branches along the discharge path from the needle tip toward the plane; these are only seen in the negative half-cycle i.e. when the needle tip is at higher potential than the plane electrode. Figure 3 shows a typical filamentary streamer. Bush-like streamers have been observed in the negative halfcycle, but filamentary streamers have never been seen in the positive half cycle.

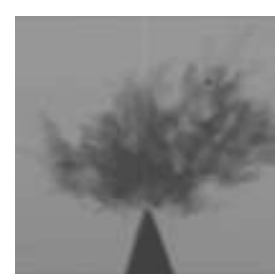

301(a)

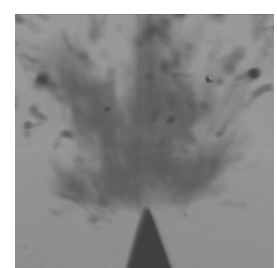

307(c)

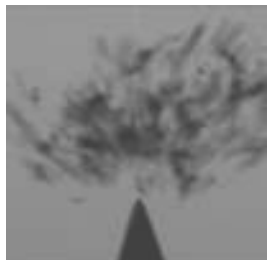

302

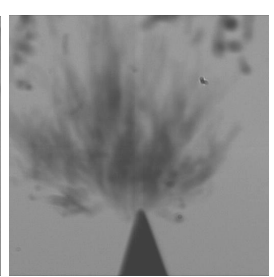

311(d)

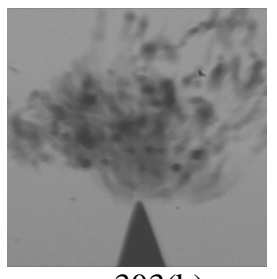

303(b)

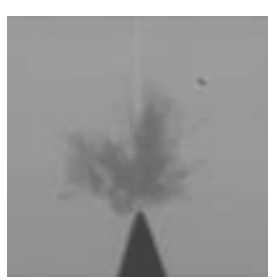

$318(\mathrm{e})$
Figure 2, Typical negative tip, bush-like discharges, with frame numbers, apparent charge and phase relates to figure 1. 


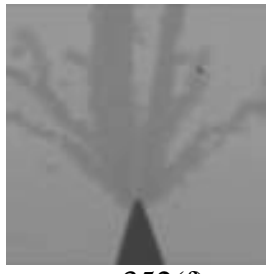

$352(\mathrm{f})$

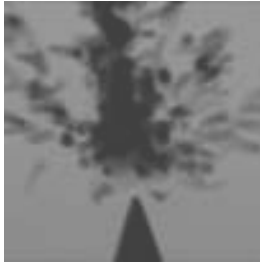

353

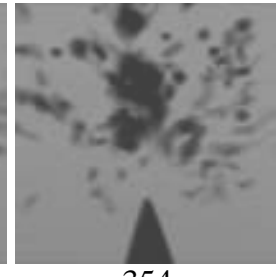

354
Figure 3, Typical positive tip filamentary discharge, with frame numbers, with apparent charge and phase related to figure 1.

\section{PTFE results}

The $\varphi \mathrm{q} n$ plots for two voltages applied to the PTFE/ $\mathrm{LN}_{2}$ composite sample are shown in Figures 4 and 5. These plots show the character of the discharges over 250 power cycles. It is evident that there is more pd activity in with the needle at lower potential than for higher, i.e. discharge is more readily initiated from a negative tip. It is also clear that there are higher maximum charge values for a positive filamentary streamer compared to the negative tip bush-like discharges, $600 \mathrm{pC}$ and $460 \mathrm{pC}$ respectively.

For the case at an applied voltage of $20.56 \mathrm{kV}$ the pd activity is grouped and centred on $105^{\circ}$ and $280^{\circ}$, with bands stretching from $45^{\circ}$ to $150^{\circ}$ and $255^{\circ}$ to $340^{\circ}$ respectively. As the voltage is increased to $26.78 \mathrm{kV}$ the activity advances to $90^{\circ}$ and $260^{\circ}$ for respective half cycles. The positive tip band also narrows to $250^{\circ}$ to $290^{\circ}$, a closer examination shows that less, large discharges occur late in the cycle. The average and maximum charge magnitudes increase for both half cycles. Average magnitudes remain at approximately $200 \mathrm{pC}$ for the negative tip and rise from $500 \mathrm{pC}$ to $550 \mathrm{pC}$ for the positive. Maximum magnitudes rise from $460 \mathrm{pC}$ to $580 \mathrm{pC}$ for the negative tip and $600 \mathrm{pC}$ to $720 \mathrm{pC}$ for the positive.

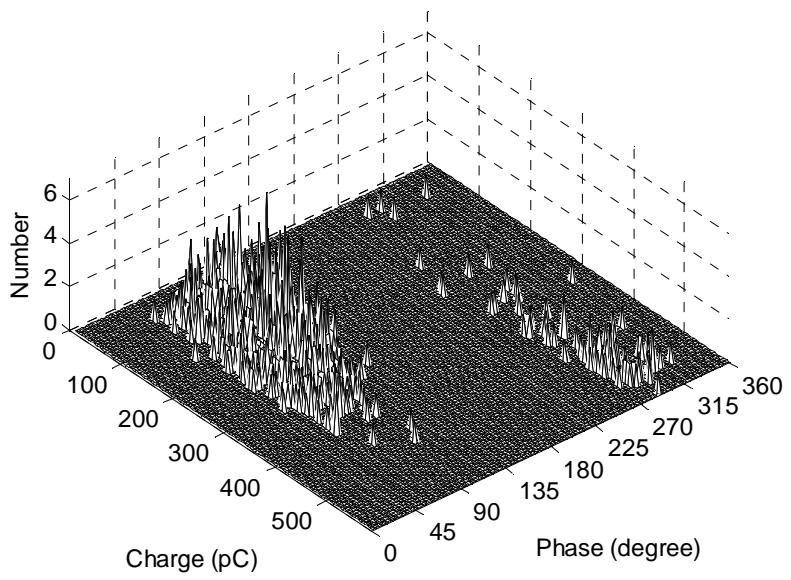

Figure 4, $\varphi \mathrm{q} \mathrm{n}$ plot with an applied voltage of $20.56 \mathrm{kV}$ at $77.3 \pm 1 \mathrm{~K}$, atmospheric pressure

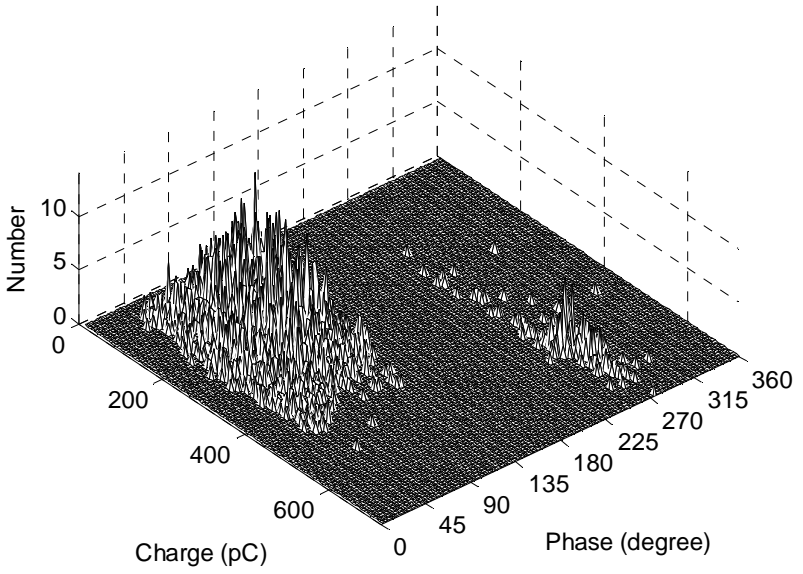

Figure 5, $\varphi \mathrm{q}$ n plot with an applied voltage of $26.78 \mathrm{kV}$ at $77.3 \pm 1 \mathrm{~K}$, atmospheric pressure

The most significant increase is in cumulate discharge numbers. For cumulative numbers by phase window an increase in the peak from 50 at $20.56 \mathrm{kV}$ to a peak of 140 at $26.78 \mathrm{kV}$ is seen, this represents a large increase in activity considering the increase across the whole activity band. It is noted that a similar trend was also seen for an increase in voltage for the $6 \mathrm{~F} / 40 \mathrm{GRR}$. A more dramatic phase advance and increase in the average and maximum discharge magnitudes was seen for the $10 \mathrm{G} / 40$ sample set with a closer gap of $2.6 \mathrm{~mm}$, further detail from this experiment can be found in [6].

\section{$6 F / 40$ results}

Figures 6 and 7 present the effect of increasing the applied pressure on the pd activity for a fixed voltage, in this example for the $6 \mathrm{~F} / 40$ sample.

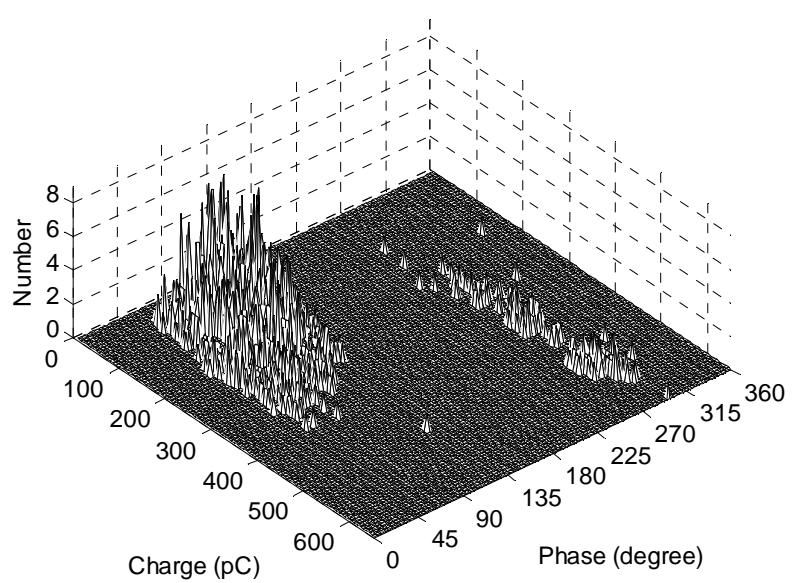

Figure 6, $\varphi \mathrm{q} \mathrm{n}$ plot with an applied voltage of $20.71 \mathrm{kV}$ at $77.4 \pm 1 \mathrm{~K}$, atmospheric pressure 


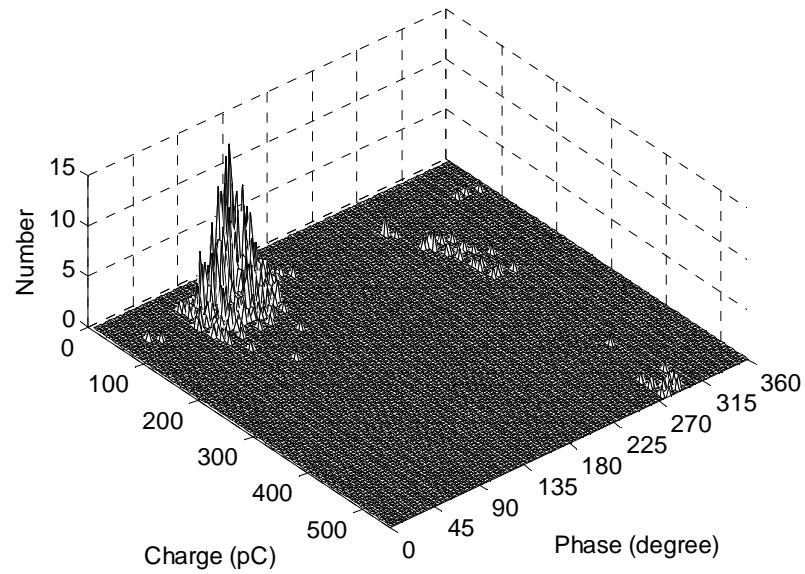

Figure 7, $\varphi$ q n plot with an applied voltage of $20.73 \mathrm{kV}$ at $77.2 \pm 1 \mathrm{~K}, 0.4 \mathrm{MPa}$,

As the pressure is increased the grouped activity is slightly retarded of the order of $5^{\circ}$ to $10^{\circ}$ in phase as the pressure is increased, this is consistent with the result for $10 \mathrm{G} / 40$. It is unclear if the distribution is skewing or retarding, whichever is the case it would indicate less charge storage in the sample. However the effect of pressurising to this level has less dramatic effect than was seen for the 10G/40 sample for a decrease in temperature [6]. Cumulative numbers by phase window are reduced for both half-cycles, Figure 7 . The increase in pressure causes the bush-like streamers from the negative tip to reduce in average and maximum charge magnitudes. The average charge drops from around 150 to $90 \mathrm{pC}$ for the negative tip and the maximums from 500 to $200 \mathrm{pC}$. It has also been observed that an increase in applied voltage to $28 \mathrm{kV}$ at $0.4 \mathrm{MPa}$ shows the same trends as for an increase in volts at a lower pressure. For the positive tip the activity band narrows, this remains unchanged for the negative tip.

At various stages in the experimental run the pd inception and extinction voltage was measured. This showed a gradual increase related to the time for which the sample had been subjected to applied voltage. This indicates the gradual erosion of the needle tip with discharge activity. After the experimental run was complete, the needles and samples were removed for inspection. This showed that the needles were eroded by the discharges.

\section{DISCUSSION AND CONCLUSIONS}

The results show that the different types of discharge hold for all samples and experimental conditions studied. It would appear that there is a difference in the mechanism of predischarge dependent on the polarity of the needle electrode. This is further supported by the observation that the positive tip pre-discharge appears to be less pressure dependant than for the negative tip.

For the negative needle tip electrons could be readily supplied by field emission from the high electric stress at the needle tip; these would be attracted to the plane electrode. Electron avalanche would propagate through the liquid phase, local heating resulting in the density change streamers seen in the images of Figure 2. The electron paths would spread due to the reducing field strength moving away from the tip and towards the plane electrode. This would also allow the charges to repel. This along with a large area from which to initiate could be responsible for the bush-like shape of the density change streamer. This would also accounts for the observed spreading of the base of the bush structure away from the needle tip as repetitive discharges continue and the initial electric field profile is distorted by the movement of charge

The average discharge magnitude is higher in the negative half-cycle when the needle tip is at a higher potential than the plane electrode. Electron scavenging must occur from the liquid nitrogen and free electrons in the liquid are not so readily available. With increasing potential eventually there is a breakdown to the needle tip, this results in space charge and leads to further propagation in to the liquid. With the electrons directed toward the needle tip this will lead to a narrow conduction path, as seen in Figure 3.

\section{REFERENCES}

1. Gerhold, J. "Dielectric breakdown of cryogenic gases and liquids", Cryogenics. 1979, pp. 571-584.

2. Hayakawa, N., Sakakibara, H., Goshima, H., Hikita, M., Okubo, H. "Breakdown mechanism of liquid nitrogen viewed from area and volume effects", IEEE Transactions on Dielectrics and Electrical Insulation. Vol. 4 No. 1, 1997, pp. 127-134.

3. Suehiro, J., Matsumoto, Y., Imasaka, K., Hara, M. "Partial discharge induced bubbles generated in subcooled liquid nitrogen at atmospheric pressure". 13th International Symposium on High Voltage Engineering (ISH), 2003. pp. 153.

4. Takahasi, Y., Ohtsuka, K. "Corona discharges and bubbling in liquid nitrogen", Journal of Physics D (Applied Physics). Vol. 8, 1974, pp. 165-169.

5. Swaffield, D. J., Lewin, P. L., Chen, G., Swingler, S. G. "Variable Pressure and Temperature Liquid Nitrogen Cryostat for Optical Measurements with Applied Electric Fields", Journal of Measurement Science and Technology. Vol. 15 No. 11, 2004, pp. 2325-2332.

6. Swaffield, D. J., Lewin, P. L., Tian, Y., Chen, G., Swingler, S. G. "Characterisation of partial discharge behaviour in liquid nitrogen". International Symposium on Electrical Insulation, 2004. IEEE, pp. 135-138. 